TRANSACTIONS OF THE

AMERICAN MATHEMATICAL SOCIETY

Volume 351, Number 12, Pages 4727-4740

S 0002-9947(99)02154-6

Article electronically published on August 27, 1999

\title{
WARPED PRODUCTS OF METRIC SPACES OF CURVATURE BOUNDED FROM ABOVE
}

\author{
CHIEN-HSIUNG CHEN
}

\begin{abstract}
In this work we extend the idea of warped products, which was previously defined on smooth Riemannian manifolds, to geodesic metric spaces and prove the analogue of the theorems on spaces with curvature bounded from above.
\end{abstract}

\section{INTRODUCTION}

The notion of warped product of Riemannian manifolds was first introduced in the paper [7]. Since then they have become an important tool for constructing examples of spaces in which the sectional curvature can be controlled but which still possess interesting topological properties. All of the classical space-times of general relativity can be presented in warped product form and this has proved important in calculating their curvature and determining maximal analytic extensions, see [18] for details. Recently, Nölker derived a decomposition theorem for an isometric immersion $h: W \rightarrow N_{\kappa}^{n}$ of a warped product $W$ into the standard $n$-space $N_{\kappa}^{n}$ of constant curvature $\kappa$ and classified all warped product decompositions of the standard spaces [17].

The warped product of two Riemannian manifolds with respect to a warping function is defined as follows: Let $M$ and $N$ be Riemannian manifolds and $f>0$ a differentiable function on $M$. Consider the differentiable manifold $M \times N$ with its projections $P_{M}: M \times N \rightarrow M$ and $P_{N}: M \times N \rightarrow N$. The warped product $W=M \times{ }_{f} N$ is the manifold $M \times N$ equipped with the Riemannian structure such that

$$
\|X\|^{2}=\left\|P_{M \star}(X)\right\|_{M}^{2}+f^{2}\left(P_{M}(m)\right)\left\|P_{N \star}(X)\right\|_{N}^{2}
$$

for every tangent vector $X \in W_{m}$.

The warped product of two Riemannian manifolds of negative curvature with convex warping function is characterized in the following two theorems.

Theorem 1.1 ([7]). Let $M$ and $N$ be Riemannian manifolds, and let $f>0$ be a differentiable function on $M$. Then the warped product $M \times_{f} N$ has curvature $K(\pi)<0$ if the following conditions hold:

1. $\operatorname{dim} M=1$, or $K<0$ on $M$.

2. $f$ is strictly convex.

3. (a) $\operatorname{dim} N=1$, or (b) $L<0$ if $f$ has a minimum; $L \leq 0$ if $f$ does not have a minimum ( $L$ is the sectional curvature of $N)$.

Received by the editors January 29, 1997.

1991 Mathematics Subject Classification. Primary 53C20, 53C21, 53C45.

(C)1999 American Mathematical Society 
Theorem 1.2 ([7]). If $M$ is complete and $W=M \times_{f} N$ has negative curvature, then conditions 1,2 , and 3 of the preceding theorem hold.

In 1951 A. D. Alexandrov introduced spaces with curvature bounded from above, which laid the foundation for a far-reaching generalization of Riemannian manifolds $[3,4]$. Alexandrov uses synthetic methods to express geometric structure in terms of inequalities on lengths of geodesics and angles between them, rather than assuming that the space has a manifold structure and computing its local geometric data. Such spaces recently have found many important applications in combinatorial group theory, analysis and even Riemannian manifolds with boundary. See $[1,13$, 14, 15] for detail.

In this work we extend the idea of warped products to geodesic metric spaces and prove analogues of the above theorems on spaces with curvature bounded from above. These results formed the core of the author's thesis [12], which also includes more details of some elementary matters. Suppose that function $f: M \rightarrow R^{+}$ is continuous and $\left(M \times_{f} N, d\right)$ denotes the warped product of two metric spaces $\left(M, d_{M}\right)$ and $\left(N, d_{N}\right)$. We prove the following theorems in this paper.

Theorem 1. If $\left(M, d_{M}\right)$ and $\left(N, d_{N}\right)$ are geodesic metric spaces and if $\left(M \times_{f} N, d\right)$ has nonpositive curvature, then

1. $\left(M, d_{M}\right)$ has nonpositive curvature.

2. $\left(N, d_{N}\right)$ has nonpositive curvature if $f$ has a minimum.

3. $f$ is convex.

Theorem 2. Let $M$ be $R$ or a countable metric graph. If $\left(N, d_{N}\right)$ has nonpositive curvature and $f: M \rightarrow R^{+}$is convex, then $\left(M \times_{f} N, d\right)$ has nonpositive curvature.

Corollary 1. Suppose that $K<0$ and $G$ is a countable metric graph. Let $f: G \rightarrow$ $R^{+}$be convex. If $\left(N, d_{N}\right)$ has curvature bounded from above by $K$, then $\left(G \times_{f} N, d\right)$ has nonpositive curvature.

Proof. This follows from Theorem 2 and the fact that if $M$ has curvature bounded from above by $K^{\prime}$, then $M$ has curvature bounded from above by $K^{\prime \prime}$, where $K^{\prime} \leq$ $K^{\prime \prime}[16]$.

The following problem is proposed by Professor Reshetnyak [20].

Let $I=\left[-\frac{\pi}{2 \sqrt{K}}, \frac{\pi}{2 \sqrt{K}}\right]$ and $f: I \rightarrow R$ be defined by $f(t)=\cos \sqrt{K} t$. If $\left(N, d_{N}\right)$ has curvature bounded from above by $K$, is $\left(I \times{ }_{f} N, d\right)$ a space of curvature bounded from above?

Using a proof similar to that of Theorem 2 the above problem is answered by the following corollary.

Corollary 2. If $\left(N, d_{N}\right)$ has curvature bounded from above by $K$, then $\left(I \times_{f} N, d\right)$ has curvature bounded from above by $K$.

The following corollary deals with spaces of negative curvature. The similar proof in Corollary 2 can be carried out in this case.

Corollary 3. Suppose $K<0$. Let $f: R \rightarrow R^{+}$be given by $f(t)=\cosh \sqrt{-K}$. If $\left(N, d_{N}\right)$ has curvature bounded from above by $K$, then $\left(R \times_{f} N, d\right)$ has curvature bounded from above by $K$. 


\section{Preliminaries}

In this section, we review relevant concepts related to this work. For more details concerning basic definitions and properties of spaces of curvature bounded from above, see $[5,6,16]$.

2.1. Curves and intrinsic metrics. A (parametrized) curve is a continuous map from an interval to a metric space. The length of curves in a metric space is defined by the usual supremum of partition sums.

A rectifiable curve is one with finite length. A rectifiable curve is said to have constant speed if the length of the restriction to any subinterval is proportional to the length of the subinterval.

Let $(M, \rho)$ be a metric space such that any pair of points of the space can be join by a rectifiable curve. We can use the metric $\rho$ to define a new metric $\rho_{\text {int }}$ which is introduced as follows:

$$
\rho_{\text {int }}(x, y)=\inf _{\gamma}\left\{l_{\rho}(\gamma)\right\}
$$

where $l_{\rho}(\gamma)$ is the length of a curve $\gamma$ in the metric $\rho$ and inf is taken over all curves joining the given pair of points $x, y$. The metric $\rho$ is said to be intrinsic if

$$
\rho=\rho_{\text {int }} .
$$

2.2. Geodesics and geodesic metric spaces. A curve in a metric space $(M, \rho)$ is called a geodesic (segment) of its length is equal to the distance between its ends. We often use $A B$ to denote $\rho(A, B)$ or the geodesic itself.

A metric space is called geodesic if for any two points in metric space, there is a geodesic between them. We do not require geodesics to have constant speed, even though that requirement is customary in Riemannian manifolds.

2.3. $S_{K}$, triangles and comparison triangles. By $S_{K}$ we denote a surface of constant curvature $K$.

A triangle $T=\triangle A B C$ in a metric space $(M, \rho)$ with vertices $A, B, C$ is the union of geodesic segments $A B, B C, A C$, called the sides of $T$.

Let $T=\triangle A B C$ be a triangle in a metric space. A triangle $T^{\prime}=\Delta A^{\prime} B^{\prime} C^{\prime}$ in $S_{K}$ is said to be a comparison triangle for $T=\triangle A B C$ if $A B=A^{\prime} B^{\prime}, B C=B^{\prime} C^{\prime}$ and $A C=A^{\prime} C^{\prime}$.

2.4. Domains $R_{K}$ and spaces of curvature bounded from above. An $R_{K}$ domain is a metric space with the following properties

1. $R_{K}$ is a geodesic space with intrinsic metric.

2. If $K>0$, then the perimeter of each triangle in $R_{K}$ is less than $2 \pi / \sqrt{K}$.

3. Each triangle in $R_{K}$ satisfies Theorem 2.1.

A space of curvature bounded from above by $K$ in the sense of A. D. Alexandrov is a space with intrinsic metric in which locally is an $R_{K}$ domain.

Theorem 2.1 ([4] $K$-concavity). Let $D, E$ be points on the sides $A B$ and $A C$ of the triangle $T=\triangle A B C$ in a domain $R_{K}$ and $D^{\prime}$ and $E^{\prime}$ the corresponding points on the sides of the comparison triangle $T^{\prime}=\Delta A^{\prime} B^{\prime} C^{\prime}$ on $S_{K}$, i.e., $A^{\prime} D^{\prime}=A D$, $A^{\prime} E^{\prime}=A E$. Then

$$
D E \leq D^{\prime} E^{\prime}
$$


We say that the metric of a metric space is $K$-concave if inequality (1) holds for an arbitrary triangle $T$ of the space.

We also need a result about gluing of spaces of curvature bounded from above by $K$.

Theorem 2.2 ([19] Gluing Theorem). If the spaces $\left(M^{\prime}, \rho^{\prime}\right)$ and $\left(M^{\prime \prime}, \rho^{\prime \prime}\right)$, which are spaces of curvature bounded from above by $K$, are glued together along compact convex sets $V^{\prime} \subset M^{\prime}$ and $V^{\prime \prime} \subset M^{\prime \prime}$ by means of some isometry $\varphi$, then the space $\left(M^{\prime} \cup_{\varphi} M^{\prime \prime}, \rho_{\varphi}\right)$ that arises as a result of gluing is also a space of curvature bounded from above by $K$.

Remark 2.1. Although Reshetnyak's theorem was originally stated, in this way, for compact convex sets, an extension to closed locally convex sets has been proved by Buyalo [11] and Bridson-Haefliger [8]. We use it in that form.

\section{Properties of NONPOSITIVEly CURVED WARPED PRODUCTS}

3.1. Definition of warped products of metric spaces. We begin this section with the definition of the length of any curve as the limit of partition sums as the partition is refined. Then we define the distance between two points as the infimum of the lengths of all curves joining these two points. The definition of warped products of metric spaces will also be given.

Suppose $\left(M, d_{M}\right)$ and $\left(N, d_{N}\right)$ are two intrinsic metric spaces. Let $\gamma=(r, s)$ : $[0,1] \rightarrow M \times N$ be a curve and $f: M \rightarrow R^{+}$be a continuous function on $M$. Suppose

$$
\tau: 0=t_{0}<t_{1}<t_{2}<\cdots<t_{n}=1 \text { is a partition of } I=[0,1] .
$$

One defines the length of $\gamma$ by

$$
l(\gamma)=\lim _{\tau} \sum_{i=1}^{n} \sqrt{d_{M}^{2}\left(r\left(t_{i-1}\right), r\left(t_{i}\right)\right)+f^{2}\left(r\left(t_{i-1}\right)\right) d_{N}^{2}\left(s\left(t_{i-1}\right), s\left(t_{i}\right)\right)} .
$$

Here the limit is taken with respect to the refinement ordering of partitions. It can be shown that the choice of evaluating $f$ at the initial point of each interval is of no consequence. Thus the distance between two points $x, y$ in $M \times N$ is defined to be

$$
d(x, y)=\inf \{l(\gamma): \gamma \text { is a curve from } x \text { to } y\} .
$$

Lemma 3.1. Let $P_{M}:(M \times N, d) \rightarrow\left(M, d_{M}\right)$ be the projection. Then $P_{M}$ is nonincreasing. In particular $d(x, y) \geq d_{M}\left(P_{M} x, P_{M} y\right)$ for any points $x$ and $y$ in $M \times N$.

Proof. For any curve $\gamma: I \rightarrow M \times N$ and any partition $\tau$, we have

$$
\begin{aligned}
& \sum_{i=1}^{n} \sqrt{d_{M}^{2}\left(P_{M} \gamma\left(t_{i-1}\right), P_{M} \gamma\left(t_{i}\right)\right)+f^{2}\left(P_{M} \gamma\left(t_{i-1}\right)\right) d_{N}^{2}\left(P_{N} \gamma\left(t_{i-1}\right), P_{N} \gamma\left(t_{i}\right)\right)} \\
& \geq \sum_{i=1}^{n} d_{M}\left(P_{M} \gamma\left(t_{i-1}\right), P_{M} \gamma\left(t_{i}\right)\right)
\end{aligned}
$$

Hence $l(\gamma) \geq l_{d_{M}}\left(P_{M} \gamma\right)$.

Proposition 3.1. $d$ is a metric in $M \times N$. 
Proof. $d(x, y) \geq 0$ and $d(x, y)=d(y, x)$ follow immediately from the definition of $d$. First, we show the triangle inequality. For any two points $x$ and $y$ in $M \times N$, by definition of $d$, there is a curve $\alpha$ with $\alpha(0)=x$ and $\alpha(1)=y$ such that $l(\alpha) \leq d(x, y)+\varepsilon$ for arbitrary $\varepsilon \geq 0$. For any two points $y$ and $z$ in $M \times N$, there is a curve $\beta$ with $\beta(0)=y$ and $\beta(1)=z$ such that $l(\beta) \leq d(y, z)+\varepsilon$ for arbitrary $\varepsilon \geq 0$. Then there is a curve $\gamma$ which is the concatenation of curves $\alpha$ and $\beta$ such that $l(\gamma) \leq d(x, y)+d(y, z)+2 \varepsilon$ for arbitrary $\varepsilon \geq 0$. Therefore $d(x, z) \leq d(x, y)+d(y, z)$.

Next, we show the nondegeneracy, i.e., $d(x, y)=0$ implies $x=y$ for all $x$ and $y$ in $M \times N$. Suppose $d(x, y)=0$; then by Lemma 3.1 we have $d_{M}\left(P_{M} x, P_{M} y\right)=0$. Hence $P_{M} x=P_{M} y$, as $d_{M}$ is nondegenerate. By definition of $d$, there is a sequence of curves $\left\{\gamma_{n}\right\}$ such that $l\left(\gamma_{n}\right)<1 / n$. Let $f$ take its minimum at $c_{n}$ along each curve $\gamma_{n}$. Then

$$
\frac{1}{n} \geq f\left(c_{n}\right) d_{N}\left(P_{N} x, P_{N} y\right) .
$$

When $n$ approaches infinity, $c_{n}$ goes to $P_{M} x$, thus $f\left(c_{n}\right)$ goes to $f\left(P_{M} x\right)$ as function $f$ is continuous. Hence $d_{N}\left(P_{N} x, P_{N} y\right)=0$ and consequently $P_{N} x=P_{N} y$, as $d_{N}$ is nondegenerate. Therefore $x=y$.

Moreover it is not difficult to see that $l(\gamma)=l_{d}(\gamma)$ for each $\gamma$, i.e., metric $d$ is intrinsic.

Definition 3.1. The warped product of $\left(M, d_{M}\right)$ and $\left(N, d_{N}\right)$ with respect to the warping function $f$ is the set $M \times N$ equipped with the intrinsic metric $d$. We denote it by $\left(M \times_{f} N, d\right)$.

Example 3.1. Every surface of revolution (not crossing the axis of revolution) is isometric to a warped product, with $M$ the generating curve, $N$ the circle of unit radius, and $f(m) / 2 \pi$ the distance from $m \in M$ to the axis of revolution.

3.2. Proof of Theorem 1. We say a function $f$ is convex on a geodesic metric space $M$, if for each constant speed geodesic $\gamma$ in $M, f \circ \gamma$ is convex, i.e., supposing $f \circ \gamma$ defined on an open interval $(a, b)$, we have $f \circ \gamma(t x+(1-t) y) \leq t f \circ \gamma(x)+$ $(1-t) f \circ \gamma(y)$ for each $x, y \in(a, b)$ and each $t, 0 \leq t \leq 1$. Similarly we can define concave functions on any geodesic metric space. We begin with some preliminary lemmas about the warped product $M \times_{f} N$.

Lemma 3.2. Geodesics in $M$ lift horizontally to geodesics in $\left(M \times_{f} N, d\right)$. Thus, $M \times\{n\}$ is totally geodesic in $\left(M \times_{f} N, d\right)$ for every $n \in N$.

Proof. Let $g$ be the geodesic joining points $a$ and $b$ in $M$. We want to show that $g \times\{n\}$ is a geodesic in $\left(M \times{ }_{f} N, d\right)$. Let $\sigma=(r, s):[0,1] \rightarrow M \times N$ be any curve from $(a, n)$ to $(b, n)$ in $\left(M \times_{f} N, d\right)$. For any partition $\tau$, we have

$$
\begin{aligned}
& \sum_{i=1}^{n} \sqrt{d_{M}^{2}\left(r\left(t_{i-1}\right), r\left(t_{i}\right)\right)+f^{2}\left(r\left(t_{i-1}\right)\right) d_{N}^{2}\left(s\left(t_{i-1}\right), s\left(t_{i}\right)\right)} \\
& \geq \sum_{i=1}^{n} d_{M}\left(r\left(t_{i-1}\right), r\left(t_{i}\right)\right) \\
& \geq d_{M}(a, b) .
\end{aligned}
$$

Hence $l(\sigma) \geq l(g)$. 
Lemma 3.3. If $p \in M$ is a local minimum point of $f$, then $(p, \beta)$ is a geodesic in $\left(M \times_{f} N, d\right)$ for every geodesic $\beta$ in $N$.

Proof. Let $\sigma=(r, s):[0,1] \rightarrow M \times N$ be any curve joining $(p, \beta(0))$ and $(p, \beta(1))$ in $\left(M \times_{f} N, d\right)$. For any partition $\tau$, we have

$$
\begin{aligned}
& \sum_{i=1}^{n} \sqrt{d_{M}^{2}\left(r\left(t_{i-1}\right), r\left(t_{i}\right)\right)+f^{2}\left(r\left(t_{i-1}\right)\right) d_{N}^{2}\left(s\left(t_{i-1}\right), s\left(t_{i}\right)\right)} \\
& \geq \sum_{i=1}^{n} f\left(r\left(t_{i-1}\right)\right) d_{N}\left(s\left(t_{i-1}\right), s\left(t_{i}\right)\right) \\
& \geq f(p) d_{N}(s(0), s(1)) \\
& =f(p) d_{N}(\beta(0), \beta(1)) .
\end{aligned}
$$

Hence $l(\sigma) \geq l(\beta)$.

Lemma 3.4. Let $\gamma:[0,1] \rightarrow M$ be a geodesic in $M$ and $f: M \rightarrow R^{+}$be a continuous function. Let $\gamma_{p}=\gamma \times\{p\}$ and $\gamma_{q}=\gamma \times\{q\}$ be two lifts of $\gamma$, where $p$ and $q$ are any points in $N$. Then

$$
\lim _{p \rightarrow q} \frac{d\left(\gamma_{p}(t), \gamma_{q}(t)\right)}{d_{N}(p, q)}=f\left(\gamma_{q}(t)\right), \quad \forall t \in[0,1] .
$$

Proof. Let $\alpha:[0,1] \rightarrow M \times N$ be the geodesic with $\alpha(0)=(\gamma(t), p)$ and $\alpha(1)=$ $(\gamma(t), q)$. Suppose $f$ takes its minimum at point $p_{\min }$ in $M$ along the curve $P_{M}(\alpha)$ and its maximum at $p_{\max }$. Then

$$
f\left(p_{\min }\right) d_{N}(p, q) \leq l(\alpha) \leq f\left(p_{\max }\right) d_{N}(p, q) .
$$

By Lemma $3.1, l_{d_{M}}\left(P_{M}(\alpha)\right)$ goes to zero as $p$ approaches $q$, and since $f$ is continuous both $p_{\min }$ and $p_{\max }$ approach $f\left(\gamma_{q}(t)\right)$.

We also need the following basic facts about $R_{0}$ domains.

Lemma 3.5. The distance function between two geodesics in an $R_{0}$ domain is convex.

Proof. The basic idea of reducing to the case of two geodesics starting at the same point by inserting a diagonal in a quadrilateral is given in Busemann [10].

Lemma $3.6([5])$. Let $(M, \rho)$ be an $R_{K}$ domain. Then the space $(M, n \rho)$ is an $R_{K_{n}}$ domain, where $K_{n}=\frac{K}{n^{2}}$.

Proof of Theorem 1. 1. Let $x$ be any point in $M$. We shall show that there is a ball centered at $x$ which is an $R_{0}$ domain. Since $\left(M \times_{f} N, d\right)$ is a space with nonpositive curvature, we can find a ball $B_{d_{M}}(x, \lambda)$ centered at $x$ with radius $\lambda>0$ such that $B_{d_{M}}(x, \lambda) \times\{p\}$ lies in some $R_{0}$ domain in $\left(M \times_{f} N, d\right)$. Let $T$ be any triangle in $B_{d_{M}}(x, \lambda)$. Then $T \times\{p\}$ is a triangle by Lemma 3.2. Hence $T$ has the $K$-concavity property 1 and $B_{d_{M}}(x, \lambda)$ is convex. Therefore $B_{d_{M}}(x, \lambda)$ is an $R_{0}$ domain and $\left(M, d_{M}\right)$ is a space of nonpositive curvature.

2. Suppose that $f$ has minimum at point $m$ in $M$. Let $x$ be any point in $N$. Since $\left(M \times{ }_{f} N, d\right)$ is a space of nonpositive curvature, there is a ball $B_{f(m) d_{N}}(x, \lambda)$ centered at $x$ with radius $\lambda>0$ such that $\{m\} \times B_{f(m) d_{N}}(x, \lambda)$ lies in some $R_{0}$ domain in $\left(M \times_{f} N, d\right)$. Let $T$ be any triangle in $B_{f(m) d_{N}}(x, \lambda)$. Then $\{m\} \times T$ is a triangle by Lemma 3.3 . Hence $T$ has the $K$-concavity property 
1 and $B_{f(m) d_{N}}(x, \lambda)$ is convex. Therefore $B_{f(m) d_{N}}(x, \lambda)$ is an $R_{0}$ domain and $\left(N, f(m) d_{N}\right)$ is a space of nonpositive curvature.

From Lemma 3.6 we see that $\left(N, d_{N}\right)$ has nonpositive curvature.

3. Because convexity is a local property, it suffices to show that $f \circ \gamma$ is convex for every small geodesic segment $\gamma$. We parameterize $\gamma$ by its length. Let $p$ and $q$ be two points in $N$ such that $\gamma \times\{p\}$ and $\gamma \times\{q\}$ lie in an $R_{0}$ domain which belongs to $\left(M \times_{f} N, d\right)$. By Lemma 3.5, we have

$$
E F \leq t A B+(1-t) C D
$$

where $x, y \in(0, l(\gamma))$ and $0 \leq t \leq 1, A=\gamma(x) \times\{q\}, B=\gamma(x) \times\{p\}$, $C=\gamma(x) \times\{q\}, D=\gamma(y) \times\{p\}, E=\gamma(t) \times\{q\}, F=\gamma(t) \times\{p\}$. Hence

$$
\frac{E F}{d_{N}(p, q)} \leq \frac{t A B}{d_{N}(p, q)}+\frac{(1-t) C D}{d_{N}(p, q)}
$$

which gives us

$$
\lim _{p \rightarrow q} \frac{E F}{d_{N}(p, q)} \leq \lim _{p \rightarrow q} \frac{t A B}{d_{N}(p, q)}+\lim _{p \rightarrow q} \frac{(1-t) C D}{d_{N}(p, q)} .
$$

Applying Lemma 3.4, we have for each $x, y \in(0, l(\gamma))$ and each $t, 0 \leq t \leq 1$

$$
f(\gamma(t x+(1-t) y)) \leq t f(\gamma(x))+(1-t) f(\gamma(y)) .
$$

Alexandrov also defined spaces with curvature bounded from below which, roughly speaking, are dual to those of curvature bounded from above [3]. Spaces with curvature bounded from below have different flavor from those of curvature bounded from above; for details see [9]. We remark that using the same method in the proof of Theorem 1 one can show that

Theorem 3.1. If $\left(M \times_{f} N, d\right)$ has nonnegative curvature, then

1. $\left(M, d_{M}\right)$ has nonnegative curvature.

2. $\left(N, d_{N}\right)$ has nonnegative curvature, if $f$ has a minimum.

3. $f$ is concave.

\section{WARPED PRODUCTS OF NONPOSITIVELY CURVED SPACES}

4.1. Existence of geodesics. We discuss the existence of geodesics in warped product spaces in this section.

Theorem 4.1. Let $\left(M, d_{M}\right)$ be a complete, locally compact intrinsic metric space and $\left(N, d_{N}\right)$ be a geodesic metric space. Let function $f: M \rightarrow R^{+}$be continuous. Given two points $x, y$ in $\left(M \times_{f} N, d\right)$, then there exists a geodesic joining $x$ to $y$.

Proof. First we show there is a midpoint between $x$ and $y$. Let $h=d(x, y) / 2$. By definition of metric $d$, there is a sequence of curves $\left\{\gamma_{n}\right\}$ parametrized on $[0,1]$ in $M \times N$ such that $l_{d}\left(\gamma_{n}\right) \leq 2 h+\frac{1}{n}$. We now pick a geodesic segment $g$ in $N$ joining $P_{N}(x)$ and $P_{N}(y)$. Replace $P_{N} \gamma_{n}$ by $g$ and parameterize it for each $n$ as follows: Let $t^{*}$ be the first point in $[0,1]$ such that $d_{N}\left(P_{N}\left(\gamma_{n}\right)\left(t^{*}\right), P_{N}\left(\gamma_{n}\right)(0)\right)=I_{d_{N}}(g)$. Set $d_{N}(g(t), g(0))=d_{N}\left(P_{N}\left(\gamma_{n}\right)(t), P_{N}\left(\gamma_{n}\right)(0)\right)$ for every $t$ in $\left[0, t^{*}\right]$ and $g(t)=P_{N}(y)$ 
for $t$ in $\left[t^{*}, 1\right]$. Then we get a new sequence of curves $\left\{\gamma_{n}^{\prime}\right\}$. We claim that $l_{d}\left(\gamma_{n}^{\prime}\right) \leq$ $l_{d}\left(\gamma_{n}\right)$. Let $\alpha=P_{M}\left(\gamma_{n}\right)$ and $\beta=P_{N}\left(\gamma_{n}\right)$. Since

$$
\begin{aligned}
d_{N}\left(g\left(t_{i-1}\right), g\left(t_{i}\right)\right) & =\left|d_{N}\left(g\left(t_{i-1}\right), g(0)\right)-d_{N}\left(g\left(t_{i}\right), g(0)\right)\right| \\
& =\left|d_{N}\left(\beta\left(t_{i-1}\right), \beta(0)\right)-d_{N}\left(\beta\left(t_{i}\right), \beta(0)\right)\right| \\
& \leq d_{N}\left(\beta\left(t_{i-1}\right), \beta\left(t_{i}\right)\right) \text { for each } i,
\end{aligned}
$$

we have

$$
\begin{aligned}
& \sum_{i=1}^{n} \sqrt{d_{M}^{2}\left(\alpha\left(t_{i-1}\right), \alpha\left(t_{i}\right)\right)+f^{2}\left(\alpha\left(t_{i-1}\right)\right) d_{N}^{2}\left(g\left(t_{i-1}\right), g\left(t_{i}\right)\right)} \\
& \leq \sum_{i=1}^{n} \sqrt{d_{M}^{2}\left(\alpha\left(t_{i-1}\right), \alpha\left(t_{i}\right)\right)+f^{2}\left(\alpha\left(t_{i-1}\right)\right) d_{N}^{2}\left(\beta\left(t_{i-1}\right), \beta\left(t_{i}\right)\right)} .
\end{aligned}
$$

The claim now follows and $l_{d}\left(\gamma_{n}^{\prime}\right) \leq l_{d}\left(\gamma_{n}\right) \leq 2 h+\frac{1}{n}$. Let $z_{n}$ in $M \times N$ be the midpoint of curve $\gamma_{n}^{\prime}$ for every $n$. Then

$$
d\left(x, z_{n}\right) \leq h+\frac{1}{2 n} \text { and } d\left(y, z_{n}\right) \leq h+\frac{1}{2 n} .
$$

Lemma 3.1 implies that sequence $\left\{P_{M} z_{n}\right\}$ lies in $\bar{B}_{d_{M}}\left(P_{M} x, h+1\right)$ which is a compact set by Lemma 4.2. Sequence $\left\{P_{N} z_{n}\right\}$ lies in the geodesic segment $g$ which is obviously a compact set. Let $a$ in $M$ and $b$ in $N$ be the accumulation points of $\left\{P_{M} z_{n}\right\}$ and $\left\{P_{N} z_{n}\right\}$, respectively. Then $(a, b)$ in $M \times N$ is a midpoint between $x$ and $y$. We now proceed by induction to define a map

$$
\gamma:\{\text { dyadic rationals in }[0,1]\} \rightarrow M \times N
$$

with properties

1. $\gamma(0)=x, \gamma(1)=y$.

2. $d(\gamma(s), \gamma(t))=2 h|s-t|$ for all $s, t$ of dyadic rationals in $[0,1]$.

We extend the map $\gamma$ by continuity to a geodesic $\gamma$ in $M \times N$.

The following lemma will be used in Lemma 4.7.

Lemma 4.1. If $M$ and $N$ are two complete intrinsic metric spaces with $M$ locally compact, then $\left(M \times{ }_{f} N, d\right)$ is also complete for every warping function $f: M \rightarrow R^{+}$.

Proof. Applying Lemma 3.1 we see $l(\alpha) \geq l\left(P_{M}(\alpha)\right)$ for any curve $\alpha$; hence $d(p, q) \geq$ $d\left(P_{M}(p), P_{M}(q)\right.$ for all $p, q$ in $M \times N$. Therefore if $\left\{\left(p_{i}, q_{i}\right)\right\}$ is a Cauchy sequence in $M \times N$, then $\left\{p_{i}\right\}$ is a Cauchy sequence in $M$. Since $M$ is complete, $\left\{p_{i}\right\}$ converges to some point in $M$. Since $\left\{\left(p_{i}, q_{i}\right)\right\}$ is a Cauchy sequence in $M \times N$, it is bounded. So $\left\{\left(p_{i}, q_{i}\right)\right\}$ lies in a ball, say $B_{d}(x, \lambda)$ of $M \times N$ for some $\lambda>0$. Hence $\left\{p_{i}\right\}$ lies in $B_{d_{M}}\left(P_{M} x, \lambda\right)$ of $M$ by Lemma 3.2. Any curve $\gamma$ with endpoints in $B_{d_{M}}\left(P_{M} x, \lambda\right)$ and its length $\leq 2 \lambda$ will lie in the ball $B_{d_{M}}\left(P_{M} x, 2 \lambda\right)$. Suppose $f \geq c>0$ on $\bar{B}_{d_{M}}\left(P_{M} x, 2 \lambda\right)$, which is compact by Lemma 4.2 . Then by Lemma 3.3 we see $d(p, q) \geq c d\left(P_{N}(p), P_{N}(q)\right)$ for $p, q \in B_{d_{M}}\left(P_{M} x, \lambda\right) \times N$. So $\left\{q_{i}\right\}$ is a Cauchy sequence in $N$ and thus converges. So sequence $\left\{\left(p_{i}, q_{i}\right)\right\}$ converges and $\left(M \times_{f} N, d\right)$ is complete.

Lemma 4.2. Suppose $(M, \rho)$ is a complete locally compact intrinsic metric space. Then the closed ball $\bar{B}_{\rho}(x, \lambda)$ is compact for all $x$ in $M$ and $\lambda \geq 0$.

Proof. See [16] for a proof. 
4.2. Proof of Theorem 2. Theorem 2 follows immediately from Theorem 4.3 which treats the case when $M$ is the real line and Corollary 4.2 which treats the case when $M$ is a graph. The idea of proof of Theorem 4.3 is to proceed as follows: first we compare $\left(R \times_{f} N, d\right)$ to the "model" space $\left(R \times_{f} R^{2}, d^{\prime}\right)$. Next we show that $\left(R \times_{f} R^{2}, d^{\prime}\right)$ is a space with curvature bounded from above under certain assumptions. Finally we verify that $\left(R \times_{f} N, d\right)$ is a space of curvature bounded from above. The proof of Corollary 4.2 is given by the same token.

The following lemma shows that geodesics in $\left(M \times_{f} N, d\right)$ project to geodesics in $N$.

Lemma 4.3. If $\sigma=(\alpha, \delta)$ is a geodesic in $\left(M \times_{f} N, d\right)$, then $\delta$ is a geodesic in $N$. However, constant-speed is not inherited.

This follows from a more general result in which we estimate how much $\gamma=(\alpha, \beta)$ is shortened when we replace $\beta$ by a geodesic $\delta$.

Lemma 4.4. Let $\gamma=(\alpha, \beta)$ be rectifiable. Suppose that $\delta$ is a geodesic in $N$ joining the ends of $\beta$. Let $f_{\min }$ be the minimum of $f$ along $\alpha$ and suppose the ratio of lengths $c=\frac{l(\beta)}{l(\delta)}>1$. Then there is a parametrization of $\delta$ such that the curve $\sigma=(\alpha, \delta)$ satisfies

$$
l^{2}(\gamma)-l^{2}(\sigma) \geq f_{\min }^{2} \cdot\left(c^{2}-1\right) l^{2}(\delta)
$$

Proof. For given $n$ we choose a partition of the curve $\gamma$ so that the defining approximation to $l(\gamma)$ has all terms equal:

$$
\begin{aligned}
& \alpha_{i}=\text { partition point of } \alpha, \beta_{i}=\text { partition point of } \beta, f_{i}=f\left(\alpha_{i-1}\right), \\
& \qquad a_{i}=d_{M}\left(\alpha_{i-1}, \alpha_{i}\right), b_{i}=d_{N}\left(\beta_{i-1}, \beta_{i}\right), \\
& l(\gamma) \approx l_{\gamma, n}=n \sqrt{a_{i}^{2}+f_{i}^{2} b_{i}^{2}}=\sum_{j=1}^{n} \sqrt{a_{j}^{2}+f_{j}^{2} b_{j}^{2}} \text { for all } i=1, \ldots, n .
\end{aligned}
$$

We choose partition points on $\delta$ so that they are spaced proportionally to $b_{i}: b_{i}=$ $c_{n} d\left(\delta_{i-1}, \delta_{i}\right)=c_{n} d_{i}$. Then since $\delta$ is a geodesic $l(\delta)=\sum d_{i}=\frac{1}{c_{n}} \sum b_{i}$, and as $n \rightarrow \infty, c_{n} \rightarrow \frac{l(\beta)}{l(\delta)}=c$. Moreover, for a fixed parametrization of $\gamma$, the discrete partitions of $\delta$, matched with the corresponding parameter values of the partitions of $\gamma$, give us a limiting parametrization of $\delta$, which is in fact the one for which the speed is proportional to that of $\beta: l(\delta \mid[s, t])=d(\delta(s), \delta(t))=l(\beta \mid[s, t])$ for all parameter subintervals $[s, t]$. By Cauchy's inequality we have the following estimate for the length squared of the curve $\sigma=(\alpha, \delta)$ :

$$
\left(\sum \sqrt{a_{i}^{2}+f_{i}^{2} d_{2}^{2}}\right)^{2} \leq \sum 1^{2} \sum\left(a_{i}^{2}+f_{i}^{2} d_{i}^{2}\right)=n \sum\left(a_{i}^{2}+f_{i}^{2} d_{i}^{2}\right)
$$


Hence

$$
\begin{aligned}
l_{\gamma, n}^{2}-\left(\sum \sqrt{a_{i}^{2}+f_{i}^{2} d_{i}^{2}}\right)^{2} & \geq n\left(\sum\left(a_{i}^{2}+f_{i}^{2} b_{i}^{2}\right)-\sum\left(a_{i}^{2}+f_{i}^{2} d_{i}^{2}\right)\right) \\
& =n \sum f_{i}^{2}\left(b_{i}^{2}-d_{i}^{2}\right) \\
& \geq n \sum f_{\min }^{2}\left(c_{n}^{2}-1\right) d_{i}^{2} \\
& \geq\left(c_{n}^{2}-1\right) f_{\min }^{2}\left(\sum d_{i}\right)^{2}, \text { by Cauchy's inequality, } \\
& =\left(c_{n}^{2}-1\right) f_{\min }^{2} l(\delta)^{2} .
\end{aligned}
$$

Letting $n \rightarrow \infty$ we get the desired inequality:

$$
l^{2}(\gamma)-l^{2}(\sigma) \geq\left(c^{2}-1\right) f_{\min }^{2} l^{2}(\delta)
$$

Lemma 4.5. 1. If $\phi: N \rightarrow N$ is an isometric map and $\gamma=(r, s)$ is a curve in $\left(M \times{ }_{f} N, d\right)$, then $l(\gamma)=l(r, \phi(s))$.

2. If $\phi: M \rightarrow M$ is an isometric map such that $f=f \circ \psi$ and $\gamma=(r, s)$ is a curve in $\left(M \times_{f} N, d\right)$, then $l(\gamma)=l(\psi(r), s)$.

Proof. We only prove 1. For any partition $\tau$, we have

$$
\begin{aligned}
& \sum_{i=1}^{n} \sqrt{d_{M}^{2}\left(r\left(t_{i-1}\right), r\left(t_{i}\right)\right)+f^{2}\left(r\left(t_{i-1}\right)\right) d_{N}^{2}\left(s\left(t_{i-1}\right), s\left(t_{i}\right)\right)} \\
& =\sum_{i=1}^{n} \sqrt{d_{M}^{2}\left(r\left(t_{i-1}\right), r\left(t_{i}\right)\right)+f^{2}\left(r\left(t_{i-1}\right)\right) d_{N}^{2}\left(\phi\left(s\left(t_{i-1}\right)\right), \phi\left(s\left(t_{i}\right)\right)\right)} .
\end{aligned}
$$

Hence $l(\gamma)=l(r, \phi(s))$.

Lemma 4.6. Let $\left(N, d_{N}\right)$ have nonpositive curvature and let $f: R \rightarrow R^{+}$be continuous. Let $\triangle A B C$ be a triangle in $\left(R \times_{f} N, d\right)$ such that $P_{N}(\triangle A B C)$ lies in an $R_{0}$ domain. Then there is a comparison triangle $\Delta A^{\prime} B^{\prime} C^{\prime}$ in $\left(R \times_{f} R^{2}, d^{\prime}\right)$ such that $A B=A^{\prime} B^{\prime}, A C=A^{\prime} C^{\prime}, B C=B^{\prime} C^{\prime}$ and $D E \leq D^{\prime} E^{\prime}$ for all comparison points $D^{\prime}, E^{\prime} \in \triangle A^{\prime} B^{\prime} C^{\prime}$ corresponding to points $D, E \in \triangle A B C$.

Proof. Let $\triangle A B C=\Delta\left(\left(a_{1}, a_{2}\right),\left(b_{1}, b_{2}\right),\left(c_{1}, c_{2}\right)\right)$ be a triangle in $\left(R \times_{f} N, d\right)$. We shall construct a comparison triangle $\triangle A^{\prime} B^{\prime} C^{\prime}$ for the triangle $\triangle A B C$ and prove that it has the $K$-concavity property 1 . By Lemma 4.3 we see that $\Delta\left(a_{2}, b_{2}, c_{2}\right)=$ $P_{N}(\triangle A B C)$ is a triangle in $N$. Let $\Delta\left(a_{2}^{\prime}, b_{2}^{\prime}, c_{2}^{\prime}\right)$ be a comparison triangle in $R^{2}$ for $\Delta\left(a_{2}, b_{2}, c_{2}\right)$. We must check that the construction of $\Delta A^{\prime} B^{\prime} C^{\prime}$ is independent of choice of $\Delta\left(a_{2}^{\prime}, b_{2}^{\prime}, c_{2}^{\prime}\right)$ and this is assured by Lemma $4.5(1)$.

We claim that $\Delta\left(\left(a_{1}, a_{2}^{\prime}\right),\left(b_{1}, b_{2}^{\prime}\right),\left(c_{1}, c_{2}^{\prime}\right)\right)$ is a comparison triangle for $\triangle A B C$. We first show that $A B=A^{\prime} B^{\prime}$. The cases that $A C=A^{\prime} C^{\prime}, B C=B^{\prime} C^{\prime}$ are treated similarly. Let $(r, s):[0,1] \rightarrow R \times{ }_{f} N$ be a geodesic from $A$ to $B$. Let $\left(r, s^{\prime}\right):[0,1] \rightarrow R \times{ }_{f} R^{2}$ be the curve joining $A^{\prime}$ and $B^{\prime}$ such that $s^{\prime}$ is the geodesic from $a_{2}^{\prime}$ to $b_{2}^{\prime}$ parameterized so that $d_{R^{2}}\left(s^{\prime}(t), s^{\prime}(0)\right)=d_{N}(s(t), s(0))$ for every $t$ in $[0,1]$. Let $\left(r^{\prime \prime}, s^{\prime \prime}\right):[0,1] \rightarrow R \times_{f} R^{2}$ be the geodesic from $A^{\prime}$ to $B^{\prime}$. Then, for any 
partition $\tau$,

$$
\begin{aligned}
& \sum_{i=1}^{n} \sqrt{d_{R}^{2}\left(r\left(t_{i-1}\right), r\left(t_{i}\right)\right)+f^{2}\left(r\left(t_{i-1}\right)\right) d_{N}^{2}\left(s\left(t_{i-1}\right), s\left(t_{i}\right)\right)} \\
& =\sum_{i=1}^{n} \sqrt{d_{R}^{2}\left(r\left(t_{i-1}\right), r\left(t_{i}\right)\right)+f^{2}\left(r\left(t_{i-1}\right)\right) d_{R^{2}}^{2}\left(s^{\prime}\left(t_{i-1}\right), s^{\prime}\left(t_{i}\right)\right)} .
\end{aligned}
$$

Therefore

$$
l((r, s))=l\left(\left(r, s^{\prime}\right)\right) \geq l\left(\left(r^{\prime \prime}, s^{\prime \prime}\right)\right) .
$$

Similarly one can show that

$$
l\left(\left(r^{\prime \prime}, s^{\prime \prime}\right)\right) \geq l((r, s)) .
$$

Combining (2) and (3) gives

$$
l((r, s))=l\left(\left(r, s^{\prime}\right)\right) \geq l\left(\left(r^{\prime \prime}, s^{\prime \prime}\right)\right) \geq l((r, s)) .
$$

This implies $A B=A^{\prime} B^{\prime}$ and we choose geodesic $\left(r, s^{\prime}\right)$ as side $A^{\prime} B^{\prime}$.

Next we show that $D E \leq D^{\prime} E^{\prime}$. Let $D=(e, f), E=(g, h), D^{\prime}=\left(e, f^{\prime}\right)$ and $E^{\prime}=$ $\left(g, h^{\prime}\right)$ where $f^{\prime}$ and $h^{\prime}$ in $P_{R^{2}}\left(\Delta A^{\prime} B^{\prime} C^{\prime}\right)$ are the comparison points corresponding to the points $f$ and $h$ in triangle $P_{N}(\triangle A B C)$. Because $P_{N}(\triangle A B C)$ lies in an $R_{0}$ domain, we have

$$
f h \leq f^{\prime} h^{\prime} .
$$

Let $\left(u, v^{\prime}\right)$ be the geodesic joining $D^{\prime}$ and $E^{\prime}$. Let $t_{0}$ be the first point in $[0,1]$ such that $d_{R^{2}}\left(v^{\prime}(0), v^{\prime}\left(t_{0}\right)\right)=f h$. We parameterize geodesic $v$ from point $f$ to point $h$ in $N$ such that $d_{N}(v(0), v(t))=d_{R^{2}}\left(v^{\prime}(0), v^{\prime}(t)\right)$ for all $t \in\left[0, t_{0}\right]$ and $v(t)=h$ for all $t \in\left[t_{0}, 1\right]$. Then, for any partition $\tau$,

$$
\begin{aligned}
& \sum_{i=1}^{n} \sqrt{d_{R}^{2}\left(u\left(t_{i-1}\right), u\left(t_{i}\right)\right)+f^{2}\left(u\left(t_{i-1}\right)\right) d_{N}^{2}\left(v\left(t_{i-1}\right), v\left(t_{i}\right)\right)} \\
& \leq \sum_{i=1}^{n} \sqrt{d_{R}^{2}\left(u\left(t_{i-1}\right), u\left(t_{i}\right)\right)+f^{2}\left(u\left(t_{i-1}\right)\right) d_{R^{2}}^{2}\left(v^{\prime}\left(t_{i-1}\right), v^{\prime}\left(t_{i}\right)\right)} .
\end{aligned}
$$

Therefore $D E=l(u, v) \leq l\left(u, v^{\prime}\right)=D^{\prime} E^{\prime}$ and the proof is complete.

Generally the limit of Riemannian metrics with curvatures $K$ bounded from above is not a space with curvature bounded above in the sense of A. D. Alexandrov. But under the following assumptions it is. The theorem also allows us to approximate a continuous warping function by a sequence of smooth functions.

Proposition $4.1([16])$. Let $\rho_{m}, m=1,2, \ldots$, be induced Riemannian metrics given on a fixed differentiable manifold $M$ so that the metrics $\rho_{m}$ define the same topology and the following conditions are realized:

1. The Riemannian space $\left(M, \rho_{m}\right)$ is complete.

2. Sectional curvatures of $\left(M, \rho_{m}\right)$ are uniformly bounded from above by $K$ :

$$
K_{\sigma}\left(M, \rho_{m}\right) \leq K \text {. }
$$

3. For any index $m$ the injectivity radius of $M$ with respect to the Riemannian metric $\rho_{m}$ satisfies the inequality:

$$
i_{m}(M) \geq i_{o}>0 .
$$


4. The metrics $\rho_{m}$ converge uniformly to the limit metric $\rho_{\infty}$ on any compact subset of $M$.

Then the limit space $\left(M, \rho_{\infty}\right)$ is a space of curvature bounded from above by $K$.

The following theorem is a generalization of the famous Hadamard-Cartan theorem which was proved in the setting of Riemannian manifolds. This theorem implies that a simply connected space of nonpositive curvature has infinite injectivity radius.

Theorem 4.2 ([2]). A locally convex, complete geodesic space has no conjugate points.

Lemma 4.7. $\left(R \times{ }_{f} R^{2}, d\right)$ has nonpositive curvature provided the function $f: R \rightarrow$ $R^{+}$is convex.

Proof. If the function $f$ is smooth, then the result follows from Theorem 1.1. If $f$ is an arbitrary continuous convex function, then we approximate $f$ by a sequence of smooth convex functions $\left\{f_{n}\right\}$. This statement can be proved by observing that the standard smoothing mollifiers preserve convexity. Then

1. Applying Lemma 4.1, we see that the Riemannian space $\left(R \times_{f_{n}} R^{2}, d_{n}\right)$ is complete for all $n$.

2. Sectional curvatures of $\left(R \times_{f_{n}} R^{2}, d_{n}\right)$ are bounded from above by 0 follows again from Theorem 1.1.

3. The Riemannian space $\left(R \times_{f_{n}} R^{2}, d_{n}\right)$ is simply connected with nonpositive curvature. Hence it has infinite injectivity radius by Theorem 4.2.

4. The metrics $d_{n}$ clearly converge uniformly to the limit metric $d$ on any compact subset of $R \times R^{2}$.

Proposition 4.1 assures that limit space $\left(R \times{ }_{f} R^{2}, d\right)$ has nonpositive curvature. We remark that every ball in $\left(R \times{ }_{f} R^{2}, d\right)$ is an $R_{0}$ domain.

Lemma 4.8. If $\left(N, d_{N}\right)$ has nonpositive curvature and $f: R \rightarrow R^{+}$is convex, then a geodesic ball $B_{d}(p, \lambda)$ in $\left(R \times{ }_{f} N, d\right)$ is convex for sufficient small $\lambda>0$ and every point $p$ in $\left(R \times_{f} N, d\right)$.

Proof. Let $\lambda$ be sufficient small such that $P_{N}\left(B_{d}(p, \lambda)\right)$ lies in an $R_{0}$ domain. Suppose any two points $x$ and $y$ lie in a geodesic ball $B_{d}(p, \lambda)$. Consider the triangle $\Delta p x y$. By Lemma 4.6 and Lemma 4.7, there is a comparison triangle $\Delta p^{\prime} x^{\prime} y^{\prime}$ in $R^{2}$ such that $p z \leq p^{\prime} z^{\prime}$, where $z$ is any point in geodesic segment $x y$ and $z^{\prime}$ in geodesic segment $x^{\prime} y^{\prime}$ is the comparison point for $z$. Since

$$
p z \leq p^{\prime} z^{\prime} \leq \max \left\{p^{\prime} x^{\prime}, p^{\prime} y^{\prime}\right\} \leq \lambda,
$$

the geodesic ball $B_{d}(p, \lambda)$ is convex.

Theorem 4.3. If $\left(N, d_{N}\right)$ has nonpositive curvature and $f: R \rightarrow R^{+}$is convex, then $\left(R \times_{f} N, d\right)$ has nonpositive curvature.

Proof. We shall show that for any point $x$ on $\left(R \times{ }_{f} N, d\right)$ there is a ball centered at $x$ which is an $R_{0}$ domain. Let $B_{d}(x, \lambda)$ be a geodesic ball centered at $x$ with sufficiently small radius $\lambda>0$ such that $P_{N}\left(B_{d}(x, \lambda)\right)$ lies in an $R_{0}$ domain. By Lemma 4.6 and Lemma 4.7, any triangle with vertexes in $B_{d}(x, \lambda)$ has the $K$-concavity property. From Lemma 4.8 , we see that $B_{d}(x, \lambda)$ is convex. Hence $B_{d}(x, \lambda)$ is an $R_{0}$ domain, therefore $\left(R \times_{f} N, d\right)$ has nonpositive curvature. 
Theorem 4.4 ([1]). Let $M$ be a Riemannian manifold with boundary B. Then the following two conditions are equivalent:

1. $M$ has curvature bounded from above by $K$ in Alexandrov's sense.

2. The sectional curvatures of the interior of $M$ and the outward sectional curvatures of the boundary $B$ do not exceed $K$ (where an outward sectional curvature of $B$ is one that corresponds to a tangent section all of whose normal curvature vectors point outward).

Corollary 4.1. Let $I=[a, b]$ be a closed interval. If $\left(N, d_{N}\right)$ has nonpositive curvature and $f: I \rightarrow R^{+}$is convex, then $\left(I \times{ }_{f} N, d\right)$ has nonpositive curvature.

Proof. The proof of this corollary takes several steps:

1. We shall show that $\left(I \times{ }_{f} R^{2}, d^{\prime}\right)$ has nonpositive curvature. Suppose that $f$ is smooth convex function, then $\left(I \times{ }_{f} R^{2}, d^{\prime}\right)$ is a manifold with two boundaries. In the interior of $\left(I \times_{f} R^{2}, d^{\prime}\right)$, it has nonpositive curvature by Theorem 1.1. We consider the following cases for the boundaries. If $a$ is minimum, then $\{a\} \times R^{2}$ is convex in $\left(I \times{ }_{f} R^{2}, d^{\prime}\right)$, thus it has sectional curvature 0 . If $b$ is local maximum, then $\{b\} \times R^{2}$ bends toward interior. Thus there is no effect on the bound for $\left(I \times_{f} R^{2}, d^{\prime}\right)$. By Theorem 4.4, $\left(I \times_{f} R^{2}, d^{\prime}\right)$ has nonpositive curvature. For arbitrary $f$, we can use the similar argument in Lemma 4.7 to draw the conclusion.

2. Lemma 4.6 is true in this case and the proof is the same.

3. Lemma 4.8 is also true in this case.

From (1), (2) and (3), we finally conclude that each point of $\left(I \times_{f} N, d\right)$ has a neighborhood which is an $R_{0}$ domain. Therefore $\left(I \times{ }_{f} N, d\right)$ has nonpositive curvature.

Corollary 4.2. Let $G$ be a countable metric graph. If $\left(N, d_{N}\right)$ has nonpositive curvature and $f: G \rightarrow R^{+}$is convex, then $\left(G \times{ }_{f} N, d\right)$ has nonpositive curvature.

Proof. This corollary is interesting mostly only for $G=$ tree, because convex functions have to be constant on closed geodesics, and if we collapse every closed geodesic in a metric graph to a point, then the result is a tree. The proof is based on Theorem 2.2 and an elementary analysis of convex functions on graphs.

If we have a convex function $f: G \rightarrow R^{+}$, then for each vertex $v$ of $G$ there is at most one edge with end $v$ on which the maximum of $f$ on that edge is at $v$. If there were two such edges, then together they would form a geodesic segment with $v$ as an interior point, but it is impossible for a convex function to have an interior maximum. We now use induction on number of edges $n$ of $G$ to see that $G \times_{f} N$ has nonpositive curvature. When $n=1$, the assertion follows from Corollary 4.1. To prove the induction step we observe that at a vertex $v$ of $G$ we view the space as the result of gluing the space together along the single edge and the sublevel set $\{x: f(x) \leq f(v)\}$ of $f$ connected to it, with one piece for each edge on which $f$ is non-decreasing from $v$ : The pieces $\{x: f(x) \leq f(v)\} \times_{f} N$ from $v$ are isometric convex subsets of $G \times_{f} N$, so Theorem 2.2 can be applied.

\section{ACKNowledgments}

The author is greatly indebted to his advisor Professor Bishop for many suggestions during the preparation of the paper. The author would also like to express his appreciation to Professor Alexander and Professor Nikolaev for their discussions. 


\section{REFERENCES}

[1] S. B. Alexander, I. D. Berg, and R. L. Bishop, Geometric curvature bounds in Riemannian manifolds with boundary, Trans. Amer. Math. Soc. 339 (1993), 703-716. MR 93m:53034

[2] S. B. Alexander and R. L. Bishop, The Hadamard-Cartan theorem in locally convex spaces, Enseign. Math. 36 (1990), 309-320. MR 92c:53044

[3] A. D. Alexandrov, A theorem on triangles in a metric space and some of its applications, Trudy Mat. Inst. Steklov. 38 (1951), 5-23. MR 14:198a

[4] A. D. Alexandrov, Über eine Verallgemeinerung der Riemannschen Geometrie, Schr. Forschungsinst. Math. 1 (1957), 33-84. MR 19:304h

[5] A. D. Alexandrov, V. N. Berestovskii, and I. G. Nikolaev, Generalized Riemannian spaces, Russian Math. Surveys 41 (1986), 1-54. MR 88e:53103

[6] V. N. Berestovskii and I. G. Nikolaev, Multidimensional generalized Riemannian spaces, In book: Encyclopaedia of Math. Sciences 70, Springer, 1993, 184-242. CMP 94:08

[7] R. L. Bishop and B. O'Neill, Manifolds of negative curvature, Tran. Amer. Math. Soc. 145 (1969), 1-49. MR 40:4891

[8] M. Bridson and A. Haefliger, Metric Spaces of Non-positive Curvature (to appear).

[9] Yu. D. Burago, M. Gromov, and G. Perelman, A. D. Aleksandrov spaces with curvatures bounded below, Russian Math. Surveys 47:2 (1992), 1-58.

[10] H. Busemann, Spaces with non-positive curvature, Acta Mathematica 80 (1948), 259-310. MR 10:623g

[11] S. Buyalo, Lecture notes on spaces of nonpositive curvature, course taught at UIUC Spring (1995).

[12] C. H. Chen, Warped products of metric spaces of curvature bounded from above, Trans. Amer. Math. Soc. (to appear). CMP 97:17

[13] M. Gromov, Hyperbolic manifolds, groups and actions, Riemann Surfaces and Related Topics (I. Kra and Maskit, eds.), Proceedings, Stony Brook 1978, Annals of Math. Studies, Number 97, Princeton University, 1981, pp. 83-213. MR 82m:53035

[14] M. Gromov, Hyperbolic groups. Essays in Group Theory (S. M. Gersten, ed.), Math. Sciences Research Institute Publications, Number 8, Springer-Verlag, New York, Berlin, Heidelberg, 1987, pp. 75-264. MR 89e:20070

[15] M. Gromov and R. M. Schoen, Harmonic maps into singular spaces and p-adic superrigidity for lattices in groups of rank one, IHES Publications Math. 76 (1992). MR 94e:58032

[16] I. G. Nikolaev, Spaces of bounded curvature, lecture notes (1995), Urbana, Illinois.

[17] S. Nölker, Isometric immersions of warped products, Differential Geometry and its Applications 6 (1996), 1-30. MR 97d:53064

[18] B. O'Neill, Semi-Riemannian geometry with applications to relativity, Academic Press, New York, 1983. MR 85f:53002

[19] Yu. G. Reshetnyak, On the theory of spaces of curvature not greater than K, Mat. Sb. 52 (1960), 789-798.

[20] Yu. G. Reshetnyak, Personal communication.

Department of Mathematics, National Changhua University of Education, Paisa Village, Changhua 50058, Taiwan, R.O.C.

E-mail address: chen@math.ncue.edu.tw 